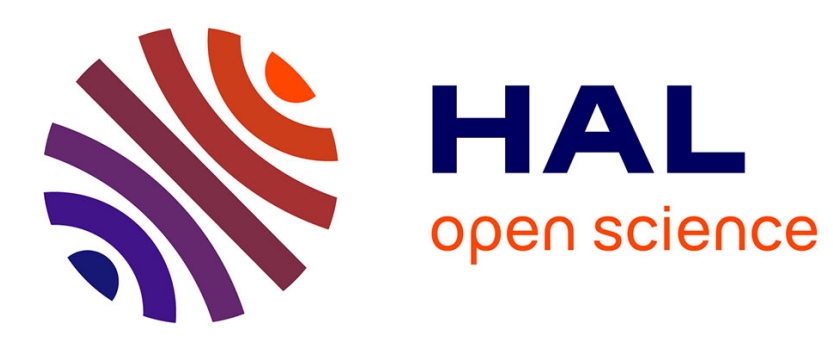

\title{
A new interval-based method to characterize estimability
}

Olivier Reynet, Luc Jaulin

\section{To cite this version:}

Olivier Reynet, Luc Jaulin. A new interval-based method to characterize estimability. International Journal of Adaptive Control and Signal Processing, 2011, 25 (3), pp.288-294. 10.1002/acs.1187 . hal-00518505

\section{HAL Id: hal-00518505 https://hal.science/hal-00518505}

Submitted on 7 Jul 2011

HAL is a multi-disciplinary open access archive for the deposit and dissemination of scientific research documents, whether they are published or not. The documents may come from teaching and research institutions in France or abroad, or from public or private research centers.
L'archive ouverte pluridisciplinaire HAL, est destinée au dépôt et à la diffusion de documents scientifiques de niveau recherche, publiés ou non, émanant des établissements d'enseignement et de recherche français ou étrangers, des laboratoires publics ou privés. 
INTERNATIONAL JOURNAL OF ADAPTIVE CONTROL AND SIGNAL PROCESSING

Int. J. Adapt. Control Signal Process. 2010; 1:1-6 Prepared using acsauth.cls [Version: 2002/11/11 v1.00]

\title{
A New Interval-Based Method to Characterize Estimability
}

\author{
O. Reynet ${ }^{1, *}$, L. Jaulin ${ }^{2}$ \\ ${ }^{1} E^{3} I^{2}$ laboratory, ENSIETA \\ 2 DTN laboratory, ENSIETA
}

\begin{abstract}
SUMMARY
Estimability is a property which states on the accuracy of the parameter estimation in the case of experimental data. This paper defines a new method based on interval analysis and set inversion to characterize estimability in the case of a bounded additive noise. To illustrate this new method, the Time Difference of Arrival (TDOA) passive location estimability is evaluated: to our knowledge, it is the first time that the parameter estimation error of these nonlinear equations is given. Copyright (C) 2010 John Wiley \& Sons, Ltd.

KEY WORDS: Estimability, Identifiability, Interval Analysis, nonlinear Models, Experimental Design.
\end{abstract}

\section{Introduction}

Estimability is a property which states on the accuracy of the parameter estimation in the case of experimental data [1, 2]. Indeed, a parameter can be identifiable [3, 4] but poorly estimable

\footnotetext{
${ }^{*}$ Correspondence to: Olivier Reynet (olivier.reynet@ensieta.fr) at $\mathrm{E}^{3} \mathrm{I}^{2}$ laboratory - ENSIETA - 2, rue F. Verny - 29806 Brest Cedex 9 - www.ensieta.fr
} 
for a given experiment. This paper defines a new method based on interval analysis and set inversion to characterize estimability in the case of a bounded additive noise.

A bounded-error estimation problem can be written under the form [5]:

$$
\mathbf{y}=\mathbf{f}(\mathbf{p})+\mathbf{e}
$$

where $\mathbf{e} \in \mathbb{E}$ stands for an additive noise vector, $\mathbb{E}$ stands for the additive noise set and $f: \mathbb{R}^{n} \rightarrow \mathbb{R}^{m}$ is a nonlinear function. Our interval-based estimability approach focuses on $\hat{\mathbf{p}}$ vectors which are estimable from $\mathbf{y}$, i.e. that can lead to the same measurement vector $\mathbf{y}$. We are looking for $\mathbb{P}$ set such as:

$$
\mathbb{P}=\left\{\hat{\mathbf{p}} \in \mathbb{R}^{n} \mid \exists\left(\mathbf{e}_{1}, \mathbf{e}_{2}\right) \in \mathbb{E}^{2}, f(\hat{\mathbf{p}})+\mathbf{e}_{1}=\mathbf{f}(\mathbf{p})+\mathbf{e}_{2}\right\}
$$

Define the uncertainty set $\mathbb{U}=\left\{\mathbf{e}_{2}-\mathbf{e}_{1} \mid \mathbf{e}_{1} \in \mathbb{E}, \mathbf{e}_{2} \in \mathbb{E}\right\}$ and $\mathbb{Y}=\mathbf{f}(\mathbf{p})+\mathbb{U}$. Then, $\mathbb{P}$ can be written as a set inversion [6]:

$$
\mathbb{P}=f^{-1}(\mathbb{Y})
$$

Figure 1 illustrates our estimability approach.

In next section, we define the estimability function $\xi_{f}$ which characterizes the size of $\mathbb{P}$. Third section shows how interval analysis and set inversion may be used to evaluate of $\xi_{f}$. Finally, last section illustrates $\xi_{f}$ relevance by evaluating the estimability of a nonlinear passive location function. 


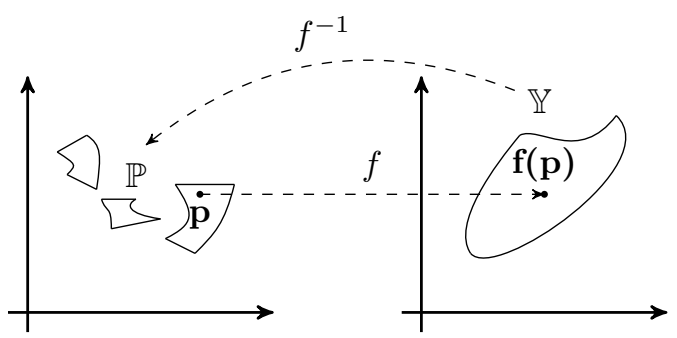

Figure 1. Illustration of our estimability approach: $\mathbb{P}$ constitutes the reciprocal image of $\mathbb{Y}=\mathbf{f}(\mathbf{p})+\mathbb{U}$.

Our estimability function $\xi_{f}$ evaluates the size of $\mathbb{P}$.

\section{Estimability Function $\xi_{f}$}

\subsection{Preliminary definition}

To define $\xi_{f}$, we need a general size function $w$ such as:

$$
\begin{array}{cl}
w: \mathcal{C}\left(\mathbb{R}^{n}\right) & \rightarrow \mathbb{R}^{+} \\
\mathbb{A} & \rightarrow w(\mathbb{A})
\end{array}
$$

where $\mathcal{C}\left(\mathbb{R}^{n}\right)$ stands for compact sets of $\mathbb{R}^{n}$. The general size function satisfies two conditions: $w(\mathbb{A})$ always belongs to $\mathbb{R}^{+}$and $w$ is monotonic, i.e. $\mathbb{A} \subseteq \mathbb{B} \Rightarrow w(\mathbb{A}) \leq w(\mathbb{B})$. Classically, $w$ is chosen as the largest dimension of the smallest box containing $\mathbb{A}$. Nevertheless, depending on the context and the dimension $n, w$ may account for area, volume or diameter of a compact set $[7,8]$.

\subsection{Estimability Function $\xi_{f}$ Definition}

In the following, $f: \mathbb{R}^{n} \rightarrow \mathbb{R}^{m}$ stands for a nonlinear function. Then we can define estimability function $\xi_{f}$ as follow: 


$$
\begin{aligned}
\xi_{f}: & \mathbb{R}^{n} \quad \rightarrow \mathbb{R}^{+} \\
\mathbf{p} & \rightarrow w\left(f^{-1}(\mathbf{f}(\mathbf{p})+\mathbb{U})\right)
\end{aligned}
$$

where $\mathbb{U}=\left\{\mathbf{e}_{2}-\mathbf{e}_{1} \mid \mathbf{e}_{1} \in \mathbb{E}, \mathbf{e}_{2} \in \mathbb{E}\right\}$ is the uncertainty set and $\mathbb{E}$ stands for the additive noise set. $\xi_{f}(\mathbf{p})$ value is the size of the inverted set of $\mathbb{Y}=\mathbf{f}(\mathbf{p})+\mathbb{U}$.

\subsection{Illustration of Estimability Function}

To illustrate $\xi_{f}$ concept, let us choose the following one-dimension nonlinear function $f$ :

$$
\begin{aligned}
f:[0,6] & \rightarrow \mathbb{R} \\
x & \rightarrow \sqrt{x} \sin (x)+x .
\end{aligned}
$$

This $f$ function is sketched in Fig. 2 and $\xi_{f}(1)$ evaluation is detailed. We suppose that the additive noise set is $[-\varepsilon / 2, \varepsilon / 2]$. Then, interval analysis allows us to write :

$$
\mathbb{U}=[-\varepsilon / 2, \varepsilon / 2]-[-\varepsilon / 2, \varepsilon / 2]=[-\varepsilon, \varepsilon] .
$$

In this example, we choose $\varepsilon=0.7$. Therefore $f^{-1}(f(1)+[-\varepsilon, \varepsilon])$ results in two intervals $\mathbb{A}_{1}$ and $\mathbb{A}_{2}$. Let us denote by $a_{i_{-}}$and $a_{i_{+}}$the $\mathbb{A}_{i}$ lower and upper bound. $w$ result is the sum of the diameters of these two intervals ${ }^{\dagger}$. That is why:

$$
\xi_{f}(1)=\left(a_{1_{+}}-a_{1_{-}}\right)+\left(a_{2_{+}}-a_{2_{-}}\right) .
$$

$\xi_{f}(1)$ is found to be about 1.55 . It characterizes parameter estimation error due to additive noise and nonlinearity of $f$ near $x=1$.

The lesser $\xi_{f}(x)$, the better the accuracy of the parameter estimation. On the contrary, $\xi_{f}(x) \gg 1$ characterizes the impossibility to properly estimate parameters: it is due to noise, low growing rate or non-injectivity of $f[9]$.

\footnotetext{
${ }^{\dagger}$ This definition of $w$ is consistent with the section 2.1 of this paper. 


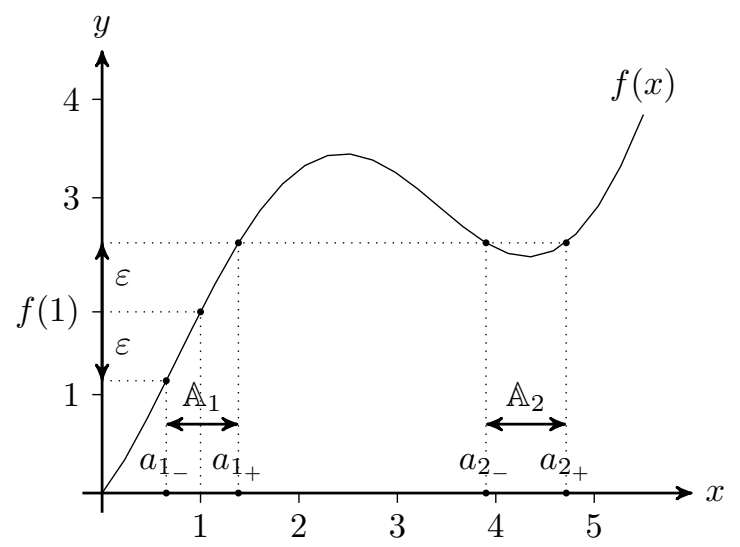

Figure 2. One dimension $f(x)=\sqrt{x} \sin (x)+x$ function and $\xi_{f}$ concept: $\varepsilon / 2=0.35, x=1$. In this example, $f^{-1}(f(1)+[-\varepsilon, \varepsilon])$ results in two intervals $\mathbb{A}_{1}$ and $\mathbb{A}_{2} \cdot \xi_{f}(1)=\left(a_{1_{+}}-a_{1_{-}}\right)+\left(a_{2_{+}}-a_{2_{-}}\right)$.

\section{Estimability Evaluation}

\subsection{Methodology}

To evaluate $\xi_{f}$, four stages are required: firstly, $\mathbb{U}$ must be deduced from $\mathbb{E}$. Secondly, $\mathbf{f}(\mathbf{p})+\mathbb{U}$ of (5) is evaluated. Then, $f^{-1}(\mathbb{Y})$ is characterized by using set inversion [10]. Finally, $w$ computes the sum of the sizes of the resulting intervals.

Powerful set methods exist to address set inversion problems [6]. In this paper, we are using Quimper, a high-level language for QUick Interval Modeling and Programming in a bounded-ERror context ${ }^{\ddagger}$. Quimper uses interval analysis and constraint propagation [11] to solve equations. It guarantees that the computed intervals enclose all solutions for given initial intervals. In addition, it provides built-in contractors which speed up computation. Details about Quimper and contractor programming can be found in [12]. But let us now illustrate

\footnotetext{
¥See Ibex/Quimper site at http://ibex-lib.org/ 
the estimability function $\xi_{f}$ on a one dimension example.

\subsection{1-D Estimability Evaluation}

$\xi_{f}$ and $f$ of (6) are drawn for $x \in[0,20]$ in Fig. 3. Each point of $\xi_{f}(x)$ has been evaluated using contractor set inversion. Quimper script for each point is similar to listing 1.

Listing 1. Example of Quimper script for set inversion

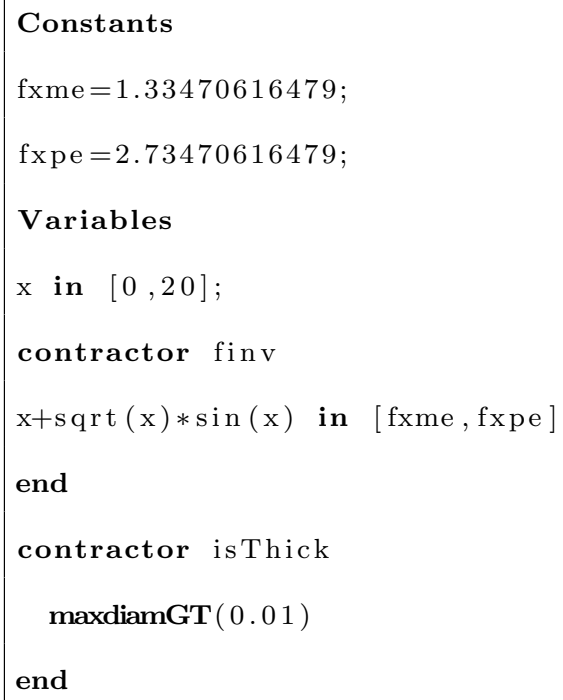

In this listing 1 , true value of $x$ is $1.1, \mathbb{E}=[-\varepsilon / 2, \varepsilon / 2]=[-0.35,0.35]$ and $[$ fxme, fxpe $]$ stands for $[f(x)-\varepsilon, f(x)+\varepsilon]$. The contractor finv eliminates all the intervals which do not satisfy (6). isThick is a special contractor to fix the bisection limits. It collects all the intervals whose maximum size is 0.01 . Therefore the intervals that are not solutions and the intervals that are indiscernible are included by contractor $i s T h i c k \S$. Computation takes about $0.002 \mathrm{~s}$ per point ${ }^{\uparrow}$.

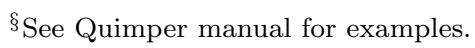

ף on an Intel Core 2 Duo CPU at $2.00 \mathrm{GHz}$

Copyright (C) 2010 John Wiley \& Sons, Ltd.

Int. J. Adapt. Control Signal Process. 2010; 1:1-6

Prepared using acsauth.cls 


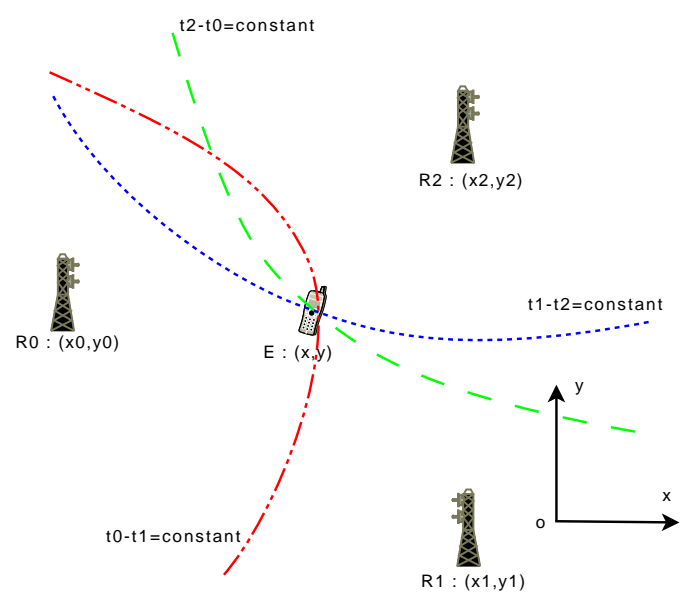

$\xi_{f}$ is not monotonic over [0,20]. Structural identifiability [9] tells us that it is due to variation of the cardinality of $f^{-1}(\mathbb{Y}) . \xi_{f}$ can take high values because of non-injectivity. On the contrary, if the injective part of $f$ is considered and if the growing rate of $f$ is high, then $\xi_{f}$ tends to 0 .

\section{Application to Passive Location}

\subsection{TDOA Hyperbolic Equations}

Let $(x, y)$ be the unknown location of the emitter, and $\left(x_{i}, y_{i}\right)$ the location of the receivers. Distance from emitter to receiver $i$ is:

$$
D_{i}=\sqrt{\left(x-x_{i}\right)^{2}+\left(y-y_{i}\right)^{2}}
$$

Let $t_{i j}$ be the measured ${ }^{\|}$Time Difference Of Arrival (TDOA) of the signal between receiver

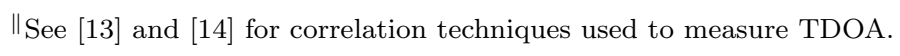



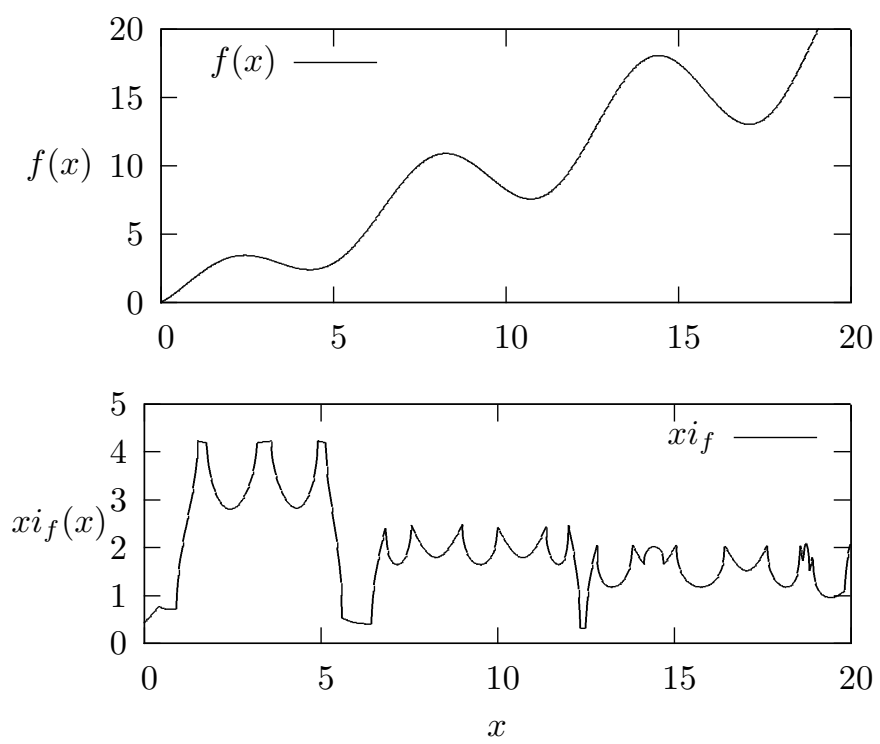

Figure 3. One dimension $f(x)=\sqrt{x} \sin (x)+x$ function and $\xi_{f}$ over x-range $[0,20]$ for $\varepsilon=0.7$.

$i$ and $j$. As $D_{i}-D_{j}=c t_{i j}$, hyperbolic TDOA equations are:

$$
\sqrt{\left(x-x_{i}\right)^{2}+\left(y-y_{i}\right)^{2}}-\sqrt{\left(x-x_{j}\right)^{2}+\left(y-y_{j}\right)^{2}}=c t_{i j}
$$

where $c$ is the speed of the signal and $(i, j) \in\{(0,1),(1,2),(2,0)\}$.

Solving these nonlinear equations for $(x, y)$ is not a trivial problem $[15,16,17]$, especially when time measurements are noisy. However, we have shown in [18] that our approach based on interval analysis, constraint propagation and contractor programming allows us to avoid any approximations and naturally results in bounded-error estimation. 


\subsection{TDOA Estimability}

Consider the following function:

$$
\begin{aligned}
f: \mathbb{R}^{2} & \rightarrow \mathbb{R}^{3} \\
(x, y) & \rightarrow\left(t_{01}, t_{12}, t_{20}\right) .
\end{aligned}
$$

where $t_{i j}$ is defined by (9). The estimability of this function allows us to refine our TDOA approach: for a given time additive noise and a special receivers configuration, we can now easily build a map which states on the TDOA passive location error.

In this example, receivers are located at R0 $(-1000,0) \mathrm{m}, \mathrm{R} 1(0,1000) \mathrm{m}$ and $\mathrm{R} 2(1000,0)$ m. We choose to define $w$ as area operator. Therefore, $\xi_{f}$ unit is $\mathrm{km}^{2}$. In this simulation, $\mathbb{E}=[-\varepsilon / 2, \varepsilon / 2] \times[-\varepsilon / 2, \varepsilon / 2] \times[-\varepsilon / 2, \varepsilon / 2]$ and $\varepsilon / 2=15$ ns. This time error** corresponds to an analog to digital converter with a good precision and a basic signal correlation.

A 100x100 $x y$ grid has been defined over x-range $[-5000,5000]$ and y-range $[-5000,5000]$. For each point $(x, y)$, a Quimper file similar to listing 2 is computed. The area corresponding to $\xi_{f}(x, y)$ is extracted from Quimper results. Figure 4 shows $\xi_{f}$ computation. Each point takes about $0.02 \mathrm{~s}$ to compute.

Listing 2. Example of Quimper script for TDOA set inversion

$$
\begin{aligned}
& \text { Constants } \\
& \mathrm{x} 0=0.0 \\
& \mathrm{y} 0=-1000.0 \\
& \mathrm{x} 1=0.0 \\
& \mathrm{y} 1=1000.0 \\
& \mathrm{x} 2=1000.0
\end{aligned}
$$

** Different $\varepsilon / 2$ could have been chosen for each $t_{i j}$. They also could have been chosen randomly. Copyright (c) 2010 John Wiley \& Sons, Ltd.

Int. J. Adapt. Control Signal Process. 2010; 1:1-6

Prepared using acsauth.cls 


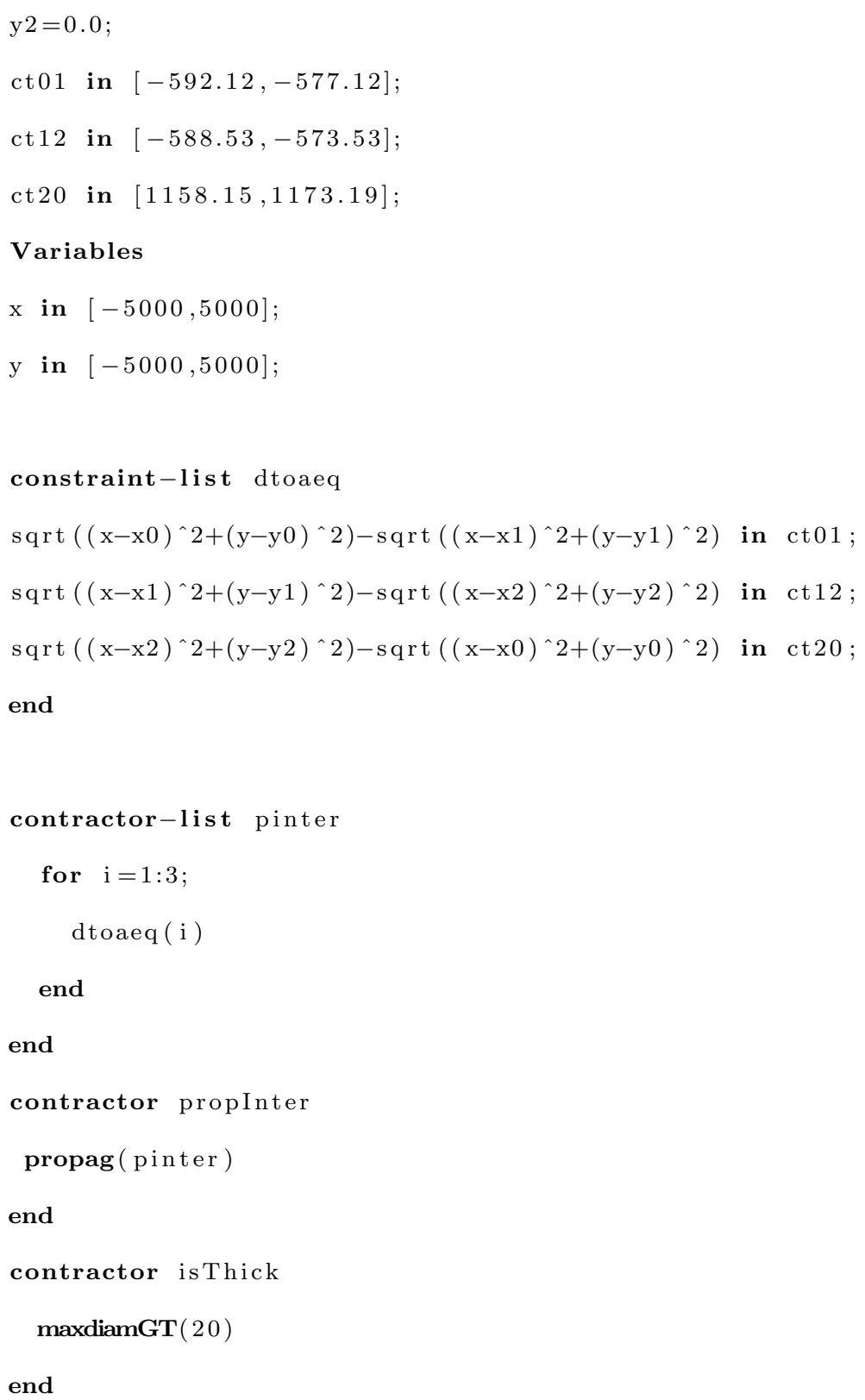

To our knowledge, it is the first time that such a function is evaluated. This map highlights the emitter positions for which the TDOA passive location error is the most important. These emitter's positions are shown to be located over complex regions really difficult to predict Copyright @ 2010 John Wiley \& Sons, Ltd. Int. J. Adapt. Control Signal Process. 2010; 1:1-6 Prepared using acsauth.cls 


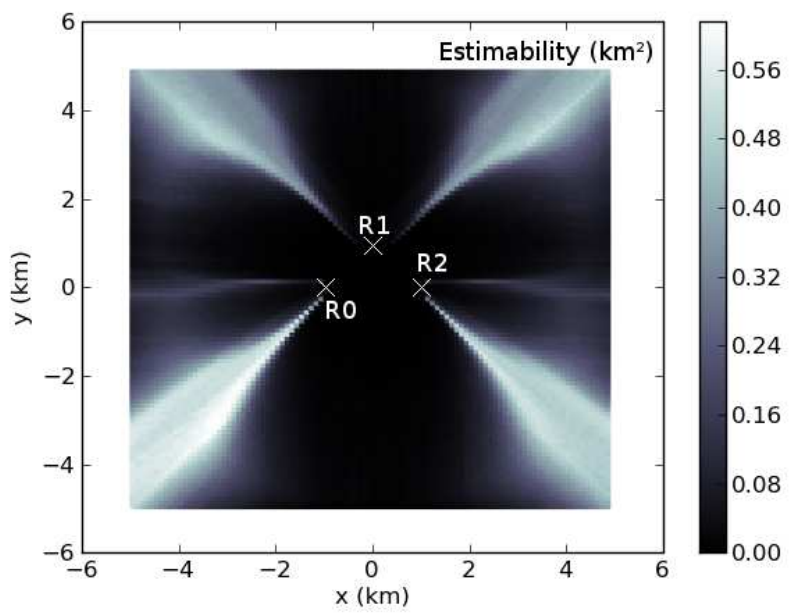

Figure 4. TDOA estimability: receivers are sketched with white crosses: R0 $(-1000,0) \mathrm{m}, \mathrm{R} 1(0,1000)$ $\mathrm{m}$ and $\mathrm{R} 2(1000,0) \mathrm{m}$.

because of the nonlinear hyperbolic equations. These intrinsic properties of $f$ are very useful to properly design passive location systems.

\section{Conclusion}

We have introduced a new interval-based method to evaluate the estimability and shown that it is possible to predict the accuracy of the parameter estimation of a nonlinear model in the case of noisy data. Our method differs from the Cramer-Rao Lower Bound (CRLB) approach, because we have not built a statistics-based estimator. Unlike CRLB, no special assumption is required on the bias or the linearity of the model, neither on the additive noise.

Our approach is not another sensitivity analysis to study the influence of the variation of the parameters on the function's result: $\xi_{f}$ directly evaluates the error of parameter estimation Copyright (c) 2010 John Wiley \& Sons, Ltd.

Int. J. Adapt. Control Signal Process. 2010; 1:1-6 Prepared using acsauth.cls 
from $f$ and additive noise set $\mathbb{E}$. Estimability function $\xi_{f}$ does not require global identifiability. Besides, its use is not restricted to small additive noise. This is due to evaluation method based on interval analysis and set inversion. Application to passive location illustrates the relevance of our approach. We are certain that numerous experimental design problems can be solved thanks to $\xi_{f}$.

\section{REFERENCES}

1. Jacquez JA, Greif P. Numerical parameter identifiability and estimability: Integrating identifiability, estimability, and optimal sampling design. MATH. BIOSCI. 1985; $\mathbf{7 7}(1-2): 201-227$. URL http://www.scopus.com/scopus/record/display.url?view=extended $\backslash \& o r i g i n=r e s u l t s l i s t \backslash \& e i d=$ 2-s2.0-0022361161.

2. Jayasankar B, Ben-Zvi A, Huang B. Identifiability and estimability study for a dynamic solid oxide fuel cell model. Computers $\&$ Chemical Engineering 2009; 33(2):484 - 492, doi:DOI:10.1016/j. compchemeng.2008.11.005. URL http://www.sciencedirect.com/science/article/B6TFT-4TYYT2-5/2/ $7 \mathrm{c} 75 \mathrm{e} 2 \mathrm{bb} 6 \mathrm{ab} 80 \mathrm{db} 40 \mathrm{~b} 6666 \mathrm{e} 8 \mathrm{ea} 155 \mathrm{c} 9 \mathrm{~b}$.

3. Ljung L, Glad T. On global identifiability of arbitrary model parametrizations. Automatica 1994; 30:265276 .

4. Berthier F, Diard JP, Pronzato L, Walter E. Identifiability and distinguishability in electrochemistry. Automatica 1996; 32:973-984.

5. Walter E, Pronzato L. Identification of Parametric Models From Experimental Data. Springer: London, 1997.

6. Jaulin L, Kieffer M, Didrit O, Walter E. Applied Interval Analysis, with Examples in Parameter and State Estimation, Robust Control and Robotics. Springer-Verlag: London, 2001.

7. Fogel E, Huang YF. On the value of information in system identification: Bounded noise case. Automatica $1982 ; \mathbf{1 8}(2): 229-238$

8. Durieu C, Walter E, Polyak B. Set-membership estimation with the trace criterion made simpler than with the determinant criterion. 12th IFAC Symposium on System Identification Sysid'2000, Cd-Rom, Santa Barbara, Californie, USA, 2000.

Copyright @ 2010 John Wiley \& Sons, Ltd.

Int. J. Adapt. Control Signal Process. 2010; 1:1-6

Prepared using acsauth.cls 
9. Lagrange S, Delanoue N, Jaulin L. Injectivity analysis using interval analysis: Application to structural identifiability. Automatica 2008; 44(11):2959 - 2962, doi:DOI:10.1016/j. automatica.2008.04.018. URL http://www.sciencedirect.com/science/article/B6V21-4TKXDBK-2/2/ c69f $4 \mathrm{e} 542 \mathrm{bdbf} 6 \mathrm{c} 5 \mathrm{cc} 0 \mathrm{~b} 6 \mathrm{e} 1 \mathrm{c} 4 \mathrm{dec} 3 \mathrm{c} 59$.

10. Jaulin L, Walter E. Set inversion via interval analysis for nonlinear bounded-error estimation. Automatica 1993; 29(4):1053-1064.

11. Jaulin L, Kieffer M, Braems I, Walter E. Guaranteed nonlinear estimation using constraint propagation on sets. International Journal of Control 2001; 74(18):1772-1782.

12. Chabert G, Jaulin L. Contractor programming. Artificial Intelligence 2009; In Press, Corrected Proof:-, doi:DOI:10.1016/j.artint.2009.03.002. URL http://www.sciencedirect.com/science/article/ B6TYF-4VVXSXC-1/2/3219355b6db4f86ba25cc09646f6899e.

13. Knapp C, Carter G. The generalized correlation method for estimation of time delay. Acoustics, Speech and Signal Processing, IEEE Transactions on Aug 1976; 24(4):320-327.

14. Carter G. Time delay estimation for passive sonar signal processing. Acoustics, Speech and Signal Processing, IEEE Transactions on Jun 1981; 29(3):463-470.

15. Chan YT, Ho KC. A simple and efficient estimator for hyperbolic location. IEEE Transactions on Signal Processing Aug 1994; 42(8):1905-1915, doi:10.1109/78.301830.

16. Foy WH. Position-location solutions by Taylor-series estimation. IEEE Transactions on Aerospace and Electronic Systems Mar 1976; 12(2):187-194, doi:10.1109/TAES.1976.308294.

17. Torrieri DJ. Statistical theory of passive location systems. IEEE Transactions on Aerospace and Electronic Systems Mar 1984; 20(2):183-198, doi:10.1109/TAES.1984.310439.

18. Reynet O, Jaulin L, Chabert G. Robust TDOA passive location using interval analysis and contractor programming. accepted to Radar 2009, 2009.

Copyright @ 2010 John Wiley \& Sons, Ltd.

Int. J. Adapt. Control Signal Process. 2010; 1:1-6

Prepared using acsauth.cls 